BULLETIN OF THE

AMERICAN MATHEMATICAL SOCIETY

Volume 79, Number 3, May 1973

\title{
TOPOLOGIES ON SPACES OF BAIRE MEASURES
}

\author{
BY R. B. KIRK \\ Communicated by R. Creighton Buck, November 6, 1972
}

Introduction. Let $X$ be a completely regular, Hausdorff space, let $C$ be the space of real-valued continuous functions on $X$ and let $C^{b}$ be the subspace of $C$ consisting of the uniformly bounded continuous functions on $X$. The Banach dual of $C^{b}$ (for the uniform norm) will be denoted by $M$, and the subspace of $M$ consisting of all totally-finite, signed Baire measures on $X$ will be denoted by $M_{\sigma}$. (Recall that the algebra of Baire sets is the smallest $\sigma$-algebra of subsets of $X$ for which each of the functions in $C$ is measurable.) Finally, the space of signed measures in $M_{\sigma}$ which have finite support will be denoted by $L$. By identifying each point of $X$ with the Dirac measure at that point we may assume that $X$ is a subset of $L$ (and hence of $M_{\sigma}$ ). The purpose of the present note is to describe some results recently obtained by the author concerning completions of $L$ relative to certain natural locally convex topologies on $L$, and some applications of these results. (For the proofs and for further details, the reader is referred to [5] and [6].) The principal results are essentially generalizations to arbitrary spaces of the following theorem due to M. Katětov and V. Ptăk. (See [3], [4] and [8].)

THEOREM. Let $X$ be pseudocompact. Then the completion of $L$ for the topology of uniform convergence on the pointwise bounded, equicontinuous subsets of $C$ is the space $M_{\sigma}(=M)$.

In order to avoid certain technical difficulties in the discussion, we will assume throughout the paper that $X$ has a nonmeasurable cardinal. (As is well known, it is consistent with the axioms of set theory to assume that there are no measurable cardinals.) For a discussion of the results in the presence of measurable cardinals, the reader is referred to [5] and [6].

1. The topology $e^{b}$. A set $B \subset C$ is equicontinuous if for all $x \in X$ and for every positive number $\varepsilon$, there is a neighborhood $U$ of $x$ such that $\mid f(x)$ $-f(y) \mid \leqq \varepsilon$ for all $y \in U$ and all $f \in B$. The set $B$ is uniformly bounded if there is a number $K$ such that $|f(x)| \leqq K$ for all $f \in B$ and all $x \in X$. Let $\mathscr{E}^{b}$ denote the family of all uniformly bounded, equicontinuous subsets of $C^{b}$; and let $e^{b}$ denote the topology on $M_{\sigma}$ of uniform convergence on the sets in $\mathscr{E}^{b}$. It is easily verified that $e^{b}$ is a locally convex topology on $M_{\sigma}$. We then have the following result:

AMS (MOS) subject classifications (1970). Primary 46A30, 60B10; Secondary 60B05.

Key words and phrases. Baire measures, weak convergence of Baire measures, complete spaces of Baire measures. 
THEOREM 1.1. Let $X$ be a completely regular Hausdorff space, and let $M_{\sigma}$ be endowed with the topology $e^{b}$. Then the following hold:

1. $M_{\sigma}$ is complete.

2. $L$ is dense in $M_{\sigma}$.

3. The dual space of $M_{\sigma}$ is $C^{b}$.

4. $X$ is a bounded subset of $M_{\sigma}$.

5. The restriction of the topology $e^{b}$ to $X$ is the original topology on $X$.

The proof of the theorem can be outlined as follows. One first considers the space $L$ with the topology $e^{b}$. It is not difficult to show that the dual of $\left(L, e^{b}\right)$ is $C^{b}$, and that, in fact, $e^{b}$ is the finest locally convex topology on $L$ whose restriction to $X$ is the original topology and for which $X$ is a bounded subset. One then shows that the completion of $\left(L, e^{b}\right)$ is $\left(M_{\sigma}, e^{b}\right)$ from which the theorem follows easily. This is done by combining the well-known theorem of A. Grothendieck characterizing complete locally convex topologies $[7$, p. 270] with certain results from the theory of measures in topological spaces.

As an application of Theorem 1.1, the following result of E. Granirer [2] may be obtained. (Of course, $M_{\sigma}^{+}$denotes the space of all finite Baire measures on $X$.)

THEOREM 1.2. Let $X$ be a completely regular Hausdorff space. Then the following hold:

1. On $M_{\sigma}^{+}$the topologies $e^{b}$ and $\sigma\left(M_{\sigma}, C^{b}\right)$ are identical.

2. If $B$ is a uniformly bounded, equicontinuous subset of $C^{b}$, then $B$ is relatively $\sigma\left(C^{b}, M_{\sigma}\right)$-compact.

It is clear that statement 2 in Theorem 1.2 is an immediate consequence of statement 3 in Theorem 1.1 and the Mackey-Arens theorem. The proof of statement 1 is somewhat more complicated. However, by using Theorem 1.1 , the analysis can be done essentially on the measures which have finite support. In this way a more elementary proof than that given by Granirer is obtained.

2. The topology $e$. A set $B \subset C$ is pointwise bounded if $\sup \{|f(x)|: f \in B\}$ is finite for all $x \in X$. Let $\mathscr{E}$ denote the family of all pointwise bounded, equicontinuous subsets of $C$. Let $M_{c}$ denote the subspace of $M_{\sigma}$ consisting of all those signed measures whose total variations have compact support in the realcompactification of $X$. (A Baire measure $m$ is said to have compact support in the realcompactification $v X$ of $X$ if there is a compact set $G$ in $v X$ such that $m(X-Z)=0$ for every zero set $Z$ in $v X$ with $G \subset Z$.) Then $M_{c}$ and $C$ may be paired by the bilinear form $\langle m, f\rangle=\int_{X} f d m$ $=\int_{X} f d m^{+}-\int_{X} f d m^{-}$. It can be verified that the topology $e$ of uniform convergence on the sets in $\mathscr{E}$ is a locally convex topology on $M_{c}$. The following can then be demonstrated. 
THEOREM 2.1. Let $X$ be a completely regular Hausdorff space, and let $M_{c}$ be endowed with the topology $e$. Then the following hold:

1. $M_{c}$ is complete.

2. $L$ is dense in $M_{c}$.

3. The dual space of $M_{c}$ is $C$.

4. The restriction of the topology $e$ to $X$ is the original topology on $X$.

In order to prove the theorem, one first shows that the topology $e$ on $L$ is the finest locally convex topology on $L$ whose restriction to $X$ is the original topology and that the dual space of $(L, e)$ is $C$. Again by combining Grothendieck's characterization of complete locally convex topologies with results from the theory of measures on topological spaces, one proves that the completion of $(L, e)$ is $\left(M_{c}, e\right)$.

An application of Theorem 2.1 yields the following analogue of Granirer's theorem (Theorem 1.2). The same method of proof is used here.

THEOREM 2.2. Let $X$ be a completely regular Hausdorff space. Then the following hold:

1. On $M_{c}^{+}$the topologies $e$ and $\sigma\left(M_{c}, C\right)$ are identical.

2. If $B$ is a pointwise bounded, equicontinuous subset of $C$, then $B$ is relatively $\sigma\left(C, M_{c}\right)$-compact.

Another application of Theorem 2.1 yields a simple proof of the following theorem of Shirota. (See [1, p. 229].)

THEOREM 2.3. Let $X$ be completely regular Hausdorff. Then there is a complete uniform structure compatible with the topology of $X$ if and only if $X$ is realcompact.

The same methods can be used to explore the closure of $L$ in other natural topologies. For instance, the completion of $L$ for the topology of uniform convergence on the norm compact subsets of $C^{b}$ is the space $M$. Also if $M_{\sigma}\left(M_{c}\right)$ is given the Mackey topology for the pair $\left(M_{\sigma}, C^{b}\right)\left(\left(M_{c}, C\right)\right)$, then it is complete and $L$ is a dense subspace. Furthermore, if $X$ is realcompact, then the completion of $L$ with the Mackey topology of the pair $(L, C)$ is $M_{c}$.

\section{REFERENCES}

1. L. Gillman and M. Jerison, Rings of continuous functions, Van Nostrand, Princeton, N.J., 1960. MR 22 \#6994.

2. E. Granirer, On Baire measures on D-topological spaces, Fund. Math. 60 (1967), 1-22. MR 34 \# 8165.

3. M. Katětov, On certain projectively generated continuity structures, Celebrazioni archimede de secolo, Simposia di topologia, 1964, pp. 47-50.

4. - Projectively generated continuity structures: A correction, Comment. Math. Univ. Carolinae 6 (1965), 251-255. MR 36 \#2110.

5. R. B. Kirk, Complete topologies on spaces of Baire measures, Trans. Amer. Math. Soc. (to appear). 
6. R. B. Kirk, Convergence of Baire measures, Pacific J. Math. (to appear).

7. G. Köthe, Topologische linear Räume. I, Die Grundlehren der math. Wissenschaften, Band 107, Springer-Verlag, Berlin, 1966; English transl., Die Grundlehren der math. Wissenschaften, Band 159, Springer-Verlag, New York, 1969. MR 33 \# 3069; MR 40 \# 1750.

8. V. Ptăk, Algebraic extensions of topological spaces, Contributions to Extension Theory of Topological Structures (Proc. Sympos., Berlin, 1967), Academic Press, New York, 1969, pp. 177-188. MR 40 \#681.

Department of Mathematics, Southern Illinois University, Carbondale, Illinois 62901 